\title{
APPLICATION OF THE MODIFIED MATERIAL POINT METHOD IN CALCULATING FLIGHT PARAMETERS
}

\author{
Dušan Regodić, \\ Damir Jerković2 \\ Aleksandar Jevremović \\ Radomir Regodić
}

\author{
${ }^{1}$ Singidunum University, \\ 32 Danijelova Street, Belgrade, Serbia \\ ${ }^{2}$ University of Defense, \\ Military Academy, \\ Pavla Jurišića Šturma 33, \\ Belgrade, Serbia
}

\section{Correspondence:}

Dušan Regodić

e-mail:

dregodic@singidunum.ac.rs

\begin{abstract}
:
The aim of this study was to analyze the effects of structural characteristics and initial conditions on foreign ballistic missile features. The problem is interesting because it extends the resolution of physical models of classical projectile motion that describes a translational movement. Applying software solutions proposed by the authors to save resources and reduce time numerical calculations as designing modern technical systems requires the use of simple physical and mathematical models and software that provide the required accuracy. The use of this software solution is in line with trends in development of modern solutions in the external ballistics. This model allows the numerical integration of equations for a shorter time than the model with six degrees of freedom.
\end{abstract}

Key words:

modified material point, force, torque, coefficients, rotate, body.

\section{INTRODUCTION}

In the classic artillery, projectile axis does not coincide with the velocity direction on the trajectory, but with it making an offensive angle $\sigma$.

Aerodynamic force $\vec{R}$ makes a certain angle with projectile axis and operates from the center of pressure (CP).

The effect of this force out of the center of mass can be replaced by force in the center of mass and moment of force according to the center of mass. This moment is called the main aerodynamic moment $\vec{M}^{R}$. Size, direction and position of attack points (center of pressure) of the total aerodynamic force depends on the size and shape of the projectile, orientation relative to the axis, velocity and angular velocity projectiles and characteristics of air.

To improve the Euler's model, we will adopt that the total aerodynamic force $\vec{R}$ consists of two dominant components, resistance $\vec{D}$ and lift $\vec{L}$, neglecting Magnusovu force (which is by intensity over 100 times smaller than the lift) and other secondary forces with unsteady character.

If we adopt the assumption that the Earth is immovable, by flight of rapid-rotary artillery projectiles, it is not necessary to take into account the influence of inertial forces, so it can be adopted $\vec{a}_{p}=\vec{a}_{c o r}=0$ and that the velocity $\vec{V}_{k}$ relative to Earth is absolute.

With the previous assumption, the vector equations of motion of mass center is reduced to 


$$
m \frac{d \vec{V}_{k}}{d t}=\sum_{i=1}^{n} \vec{F}_{i}=m \vec{g}+\vec{R}
$$

where the total aerodynamic force is $[9,10]$ :

$$
\vec{R}=\vec{D}+\vec{L},
$$

so:

$$
m \frac{d \vec{V}_{k}}{d t}=m \vec{g}+\vec{D}+\vec{L}
$$

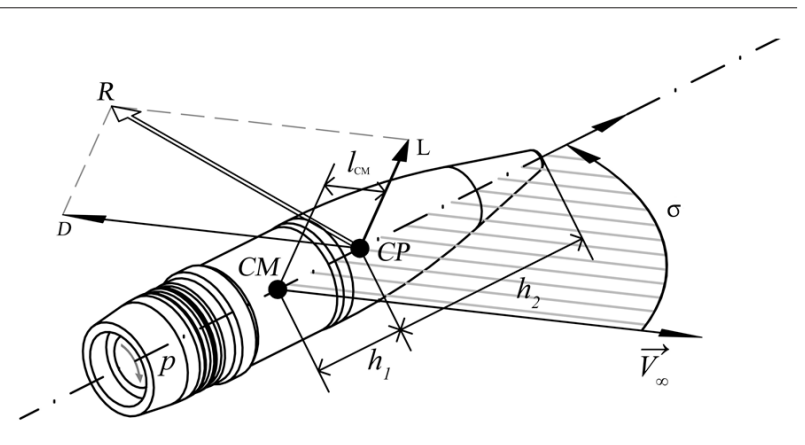

Figure 1. Air resistance and lift forces

Artillery projectile is a solid body with rotating shape. Unit vector $\vec{x}_{0}$ is the axis of rotational symmetry with the positive direction from the center of mass to the top of the projectile.

The total moment of projectile motion amount can be expressed as the sum of two vectors in a coordinate system related to the Earth:

- moment of motion amount around vectors $\vec{x}_{0}$ $I_{x} p$

- moment of motion amount around the axis normal to the vector.

Angular velocity of projectile around the axis normal to $\vec{x}_{0}$ is given by vector $\vec{\Omega}=\left(\vec{x}_{0} \times \dot{\vec{x}}_{0}\right)$. Since the projectile has a rotational symmetry, each axis that passes through the center of mass, and normal on $\vec{x}_{0}$, it is the main axis of inertia. If we check the moment of projectile inertia around some transversal axis that passes through the center of mass with $I_{y}$, the total moment of motion amount around the axis normal to $\vec{x}_{0}$ is represented by vector $I_{y}\left(\vec{x}_{0} \times \dot{\vec{x}}_{0}\right)$. The total moment of motion amount is $[1,9]$ :

$$
\vec{K}=I_{x} p \vec{x}_{0}+I_{y}\left(\vec{x}_{0} \times \dot{\vec{x}}_{0}\right)
$$

The sum of all external moments affecting the projectile is:

$$
\frac{d \vec{K}}{d t}=I_{x} \frac{d p}{d t} \vec{x}_{0}+I_{x} p \dot{\vec{x}}_{0}+I_{y}\left(\vec{x}_{0} \times \ddot{\vec{x}}_{0}\right)=\sum \vec{M} .
$$

\section{AERODYNAMIC FORCES AND MOMENTS}

The resistance force of air $\vec{D}$ represented by vector is:

$$
\vec{D}=\frac{\rho V^{2}}{2} S C_{D} \vec{V}
$$

Because of the occurrence of angle of attack $\sigma$ in the obstruction plane between $\vec{V}$ and $\vec{x}_{0}$ increases the resistance force of air due to the occurrence of induced resistance. Coefficient of induced resistance is indicated as $C_{D} \sigma^{2}$, and increase of resistance coefficient due to asymmetrical obstruction with $C_{D \sigma^{2}} \sigma^{2}$. Effective resistance coefficient is:

$$
C_{D}=C_{D 0}+C_{D \sigma^{2}} \sigma^{2}
$$

The resistance force of air is the direction $-\vec{V}$ and after the introduction of the reference surface $S=\frac{\pi d^{2}}{4}$ is:

$$
\vec{D}=-\rho \frac{\pi d^{2}}{8}\left(C_{D 0}+C_{D \sigma^{2}} \sigma^{2}\right) V \vec{V} .
$$

Lift force $\vec{L}$ occurs in the plane comprising the projectiles axis and aerodynamic velocity due to asymmetrical obstruction under the offensive angle. The direction is perpendicular to the wind velocity and proportional to the offensive corner.

$$
\vec{L}=\rho \frac{\pi d^{2}}{8} C_{L \sigma} V^{2} \sin \sigma=\rho \frac{\pi d^{2}}{8} C_{L \sigma} \sigma\left[\vec{V} \times\left(\vec{x}_{0} \times \vec{V}\right)\right]
$$

Vector $\left[\vec{V} \times\left(\vec{x}_{0} \times \vec{V}\right)\right]$ has the intensity $V^{2} \sin \sigma$ and it is normal on $\vec{V}$, and it flies in a plane that contains $\vec{V}$ and $\vec{x}_{0}$. If the vector of total aerodynamic force $\vec{R}$ is projected parallel and normal to $\vec{x}_{0}$, instead of $\vec{V}$, than we will get the components of axial force $\vec{X}$ and normal force $\vec{Y}$.

Figure 2 shows decomposition of the total aerodynamic force $\vec{R}$ on the components $\vec{X}$ and $\vec{Y}$.

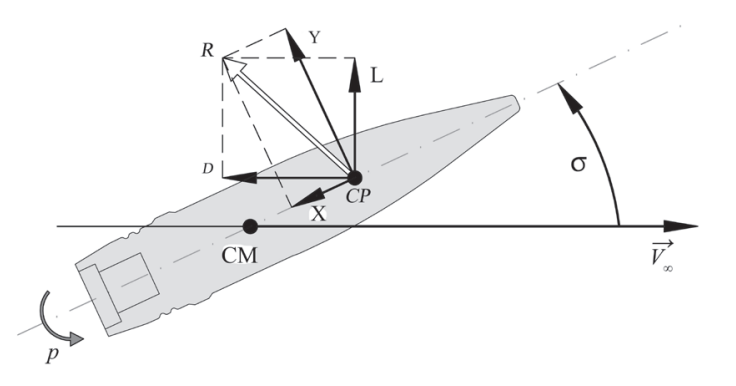

Figure 2. Dissolution of the total aerodynamic force 
The values of these forces can be written in the following form

$$
\begin{aligned}
& \vec{X}=\frac{\rho V^{2}}{2} S C_{x}=\rho \frac{\pi d^{2}}{8} V^{2} C_{x}, \\
& \vec{Y}=\frac{\rho V^{2}}{2} S C_{y}=\rho \frac{\pi d^{2}}{8} V^{2} C_{y} .
\end{aligned}
$$

Longitudinal damping moment $M_{x}$ occurs as a result of longitudinal friction over the surface of the rotating projectile and has a tendency to reduce (stop) axial rotation. The intensity of this moment is defined as:

$$
M_{x}=\frac{\rho V^{2}}{2} s C_{\ell p} p V=-\rho \frac{\pi d^{4}}{16} C_{\ell p} p V \vec{x}_{0},
$$

\section{Where is:}

$$
\begin{aligned}
& C_{\ell p} \text { - dimension less gradient coefficient of suppres- } \\
& \text { sive longitudinal moment for reference angular } \\
& \text { velocity } p^{*}=\frac{p d}{2 V} .
\end{aligned}
$$

\section{FORMATION OF THE FINAL SYSTEM OF EQUATIONS}

To resolve the lift force, it is necessary offensive angle $\sigma$ and unit vector of lift force $\vec{i}_{L}$ :

$$
L=\rho \frac{\pi d^{2}}{8} C_{L \sigma} \sigma \vec{i}_{L}
$$

If we introduce velocity coordinate system $(V)$ with the origin of coordinates at the center of projectile mass, axis $x_{V}$ in the direction of aerodynamic velocity, and $z_{V}$ in the vertical plane on the aerodynamic velocity pointing downward.

Separation of aerodynamic velocity on two components along the projectile axis and normal to aerodynamic velocity. The second component is $V \sigma \vec{i}_{L}$. Its components that coordinate the system along the axis of velocity are:

$$
[0-\tilde{\beta} V-\tilde{\alpha} V]^{T}
$$

Projection of the lift force of axis of velocity coordinate system:

$$
L^{V}=\frac{\rho V}{2} C_{L \sigma}[0-\tilde{\beta} V-\tilde{\alpha} V]^{T}
$$

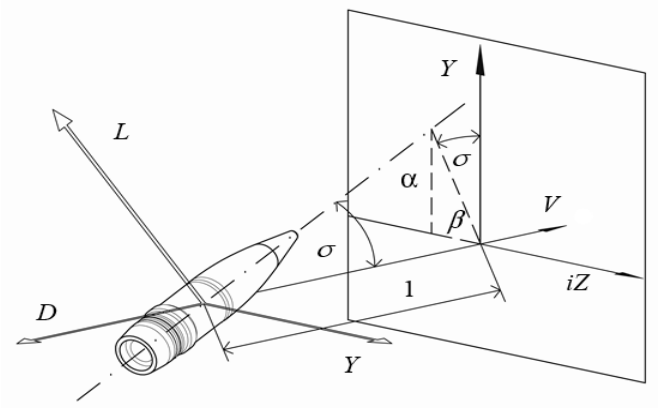

Figure 3. Projection of attack angle in space $[1,10]$

To determine the components along the local $(L)$ coordinate system, it is necessary to determine the matrix of transformation from velocity system in the nosen system. The axes of nosen coordinate system are parallel to the axes of local coordinate system. By rotation for angle $\chi_{a}$ around the axis $z$ and for angle $\gamma_{a}$ around the axis $y$ we come from the tractive to the velocity coordinate system. That matrix of transformation is $[1,2,10]$ :

$$
L_{V 0}=L_{y}\left(\gamma_{a}\right) L_{z}\left(\chi_{a}\right)
$$

According as:

$$
\begin{gathered}
L_{Z}\left(\chi_{a}\right)=\left[\begin{array}{ccc}
\cos \chi_{a} & \sin \chi_{a} & 0 \\
-\sin \chi_{a} & \cos \chi_{a} & 0 \\
0 & 0 & 0
\end{array}\right]=\left[\begin{array}{ccc}
\frac{u}{\sqrt{V^{2}-v^{2}}} & \frac{w}{\sqrt{V^{2}-v^{2}}} & 0 \\
-\frac{w}{\sqrt{V^{2}-v^{2}}} & \frac{u}{\sqrt{V^{2}-v^{2}}} & 0 \\
0 & 0 & 1
\end{array}\right] \\
L_{y}\left(\gamma_{a}\right)=\left[\begin{array}{ccc}
\cos \gamma_{a} & 0 & -\sin \gamma_{a} \\
0 & 1 & 0 \\
\sin \gamma_{a} & 0 & \cos \gamma_{a}
\end{array}\right]=\left[\begin{array}{ccc}
\frac{\sqrt{V^{2}-v^{2}}}{V} & 0 & -\frac{v}{V} \\
0 & 1 & 0 \\
\frac{v}{V} & 0 & \frac{\sqrt{V^{2}-v^{2}}}{V}
\end{array}\right]
\end{gathered}
$$

multiplying these matrices we obtain the required matrix of transformation.

Projections of vector $\sigma \vec{i}_{L}$ on axes of transmission coordinate system are:

$$
\begin{aligned}
& L_{O V}\left[\begin{array}{c}
0 \\
-\tilde{\beta} \\
-\tilde{\alpha}
\end{array}\right]=\left[\begin{array}{ccc}
\frac{u}{V} & -\frac{w}{\sqrt{V^{2}-v^{2}}} & \frac{u v}{V \sqrt{V^{2}-v^{2}}} \\
\frac{w}{V} & \frac{u}{\sqrt{V^{2}-v^{2}}} & \frac{v w}{V \sqrt{V^{2}-v^{2}}} \\
-\frac{v}{V} & 0 & \frac{\sqrt{V^{2}-v^{2}}}{V}
\end{array}\right]\left[\begin{array}{c}
0 \\
-\tilde{\beta} \\
-\tilde{\alpha}
\end{array}\right] \\
& \tilde{\alpha}=\tilde{\alpha}_{h}+\tilde{\alpha}_{p} \\
& \tilde{\beta}=\tilde{\beta}_{h}+\tilde{\beta}_{p}
\end{aligned}
$$




$$
\tilde{\beta}_{h}+i \tilde{\alpha}_{h}=C_{1} e^{r_{1} \bar{s}}+C_{2} e^{r_{2} \bar{s}}
$$

and particular:

$$
\tilde{\beta}_{p}+i \tilde{\alpha}_{p}=-\frac{G_{\xi}}{M_{\xi}+i P T_{\xi}} .
$$

The solutions of these two parts are different. Homogeneous integral is random and particular deterministic size. By initial conditions $\tilde{\beta}_{0}+i \tilde{\alpha}_{0}=0$ of the complex constants are equal and with opposite sign $C_{1}=-C_{2}$ depending on the transversal angular velocity:

$$
C_{1}=\frac{\tilde{\xi}_{0}{ }^{\prime}}{r_{1}-r_{2}}=\frac{\sigma_{0}{ }^{\prime} e^{i\left(\varphi_{0}-\phi_{0}\right)}}{r_{1}-r_{2}} .
$$

Initial angular velocity has a Rayleigh distribution, and the angle that determines the position of the plane in which is that angular velocity $\left(\varphi_{0}-\phi_{0}\right)$ relative to the vertical plane, has an equal distribution from 0 to $2 \pi$. Based on stated, we conclude that the influence is random size which is probable for all directions around velocity. Therefore, the influence of homogeneous integrals $\tilde{\xi}_{h}=\tilde{\beta}_{h}+i \tilde{\alpha}_{h}$ in the model modified material point does not take into consideration by calculating the trajectory, but it is thought to lead to distribution over trajectory for which the homogeneous integral is equal to zero.

$$
\begin{aligned}
& G_{\xi}=\frac{g d \cos \gamma}{V^{2}}\left[P-i\left(\frac{V^{\prime}}{V}+\frac{C_{\bar{m} q}^{*}}{\bar{r}_{y}^{2}}\right)\right] \approx \frac{g d \cos \gamma}{V^{2}} P \\
& M_{\xi}+i p T_{\xi} \approx M_{\xi} \approx M=\frac{C_{\bar{m} \sigma}^{*}-C_{\bar{m} \dot{\sigma}}^{*} \frac{V^{\prime}}{V}-C_{\bar{m} q}^{*} C_{\bar{z} \sigma}^{*}}{\bar{r}_{y}^{2}} \approx \frac{C_{\bar{m} \sigma}^{*}}{\bar{r}_{y}^{2}}
\end{aligned}
$$

For that case is:

$$
\tilde{\beta}_{p}+i \tilde{\alpha}_{p}=-\frac{G_{\xi}}{M_{\xi}}=-\frac{\frac{g d \cos \gamma}{V^{2}} \frac{I_{x}}{I_{y}} \frac{p d}{V}}{\frac{\rho S d}{2 m} \frac{C_{\bar{m} \sigma}}{r_{y}^{2}} d^{2}}
$$

$$
\begin{gathered}
\tilde{\alpha}_{p}=0 \\
\tilde{\beta}_{p}=-\frac{2 I_{x}}{\rho S d} \frac{g \cos \gamma}{V^{3}} \frac{p}{C_{\bar{m} \sigma}}
\end{gathered}
$$

For the calculation of particular integrals $\beta_{p}$ it is required, angular velocity $p$ for each point of the trajectory is requiried. We can determine it based on the equation of rolling aircraft.

$$
I_{x} \frac{d p}{d t}=\frac{\rho V^{2}}{2} S d C_{\mathrm{l} p} p^{*} .
$$

Required projection of vector $\sigma \overrightarrow{i_{L}}$ on the axes of tractive coordinate system:

$$
L_{O V}\left[\begin{array}{c}
0 \\
-\tilde{\beta} \\
-\tilde{\alpha}
\end{array}\right]=\left[\begin{array}{c}
\tilde{\beta}_{p} \frac{w}{\sqrt{V^{2}-v^{2}}} \\
-\tilde{\beta}_{p} \frac{u}{\sqrt{V^{2}-v^{2}}} \\
0
\end{array}\right]
$$

Components of lift force on the axis of local coordinate system are:

$$
\left[\begin{array}{c}
L x \\
L y \\
L z
\end{array}\right]=\frac{\rho V^{2}}{2} S C_{L \sigma} \tilde{\beta}_{p}\left[\begin{array}{c}
\frac{w}{\sqrt{V^{2}-v^{2}}} \\
0 \\
-\tilde{\beta}_{p} \frac{u}{\sqrt{V^{2}-v^{2}}}
\end{array}\right]
$$

Therefore, we have the system of equations modified model of material point (without Coriolis acceleration):

$$
\begin{aligned}
& \frac{d u_{k}}{d t}=-E\left(C_{D} \frac{u_{k}-u_{w}}{V}+C_{L \sigma} \tilde{\beta}_{p} \frac{w_{k}-w_{w}}{\sqrt{V^{2}-v^{2}}}\right) \\
& \frac{d v_{k}}{d t}=-E\left(C_{D} \frac{v_{k}}{V}\right)-g \\
& \frac{d w_{k}}{d t}=-E\left(C_{D} \frac{w_{k}-w_{w}}{V}-C_{L \sigma} \tilde{\beta}_{p} \frac{u_{k}-u_{w}}{\sqrt{V^{2}-v^{2}}}\right) \\
& \frac{d p}{d t}=\frac{S d^{2}}{4 I_{x}} \rho V C_{\ell p} p \\
& \frac{d x}{d t}=u_{k} \\
& \frac{d y}{d t}=v_{k} \\
& \frac{d z}{d t}=w_{k},
\end{aligned}
$$

where are:

$$
\begin{aligned}
& E=\frac{\rho V^{2}}{2} \frac{S}{m}=\rho \frac{\pi d^{2} V^{2}}{8 m}, \\
& V=\sqrt{\left(u_{k}-u_{w}\right)^{2}+v_{k}^{2}+\left(w_{k}-w_{w}\right)^{2}}, \\
& \tilde{\beta}_{p}=-\frac{2 I_{x}}{\rho S d} \frac{g \cos \gamma}{V^{3}} \frac{p}{C_{\bar{m} \sigma}} .
\end{aligned}
$$

We must add the following to the equations:

- aerodynamic functions $C_{D}(M a), C_{L \sigma}(M a)$, $C_{\bar{m} \sigma}(M a)$, and $C_{\ell p}(M a)$,

- constants $d, m$ i $I_{X}$,

- data on the state of the atmosphere $\rho(y), a(y)$ and data on wind $u_{w}(y), w_{w}(y)$,

- Initial conditions $y_{0}$ (always take $x_{0}=z_{0}=0$ ), $V_{k 0}, \gamma_{k 0}, \chi_{k 0}$, and $p_{0}$. 
Based on the stated we can add Coriolis force in the model of the modified material point:

$$
\frac{d V_{k}}{d t}=\frac{\rho V^{2}}{2} \frac{S}{m} C_{D} \frac{\vec{V}_{k}-\vec{V}_{w}}{V}+m \vec{g}+\frac{\rho V^{2}}{2} \frac{S}{m} C_{L \sigma} \sigma \vec{i}_{L}-\vec{a}_{K}
$$

Components on the axes of local coordinate system give the system of differential equations models MMMT $[1,2,4,5,9]$ :

$$
\begin{aligned}
& \frac{d u_{k}}{d t}=-E\left(C_{D} \frac{u_{k}-u_{w}}{V}+C_{L \sigma} \tilde{\beta}_{p} \frac{w_{k}-w_{w}}{\sqrt{V^{2}-v^{2}}}\right)-2\left(-\Omega_{E Z} v_{k}+\Omega_{E Y} w_{k}\right) \\
& \frac{d v_{k}}{d t}=-E\left(C_{D} \frac{v_{k}}{V}\right)-g-2\left(\Omega_{E Z} u_{k}-\Omega_{E X} w_{k}\right) \\
& \frac{d w_{k}}{d t}=-E\left(C_{D} \frac{w_{k}-w_{w}}{V}-C_{L \sigma} \tilde{\beta}_{p} \frac{u_{k}-u_{w}}{\sqrt{V^{2}-v^{2}}}\right)-2\left(-\Omega_{E Y} u_{k}+\Omega_{E X} v_{k}\right) \\
& \frac{d p}{d t}=\frac{S d^{2}}{4 I_{x}} \rho V C_{\ell p} p \\
& \frac{d x}{d t}=u_{k} \\
& \frac{d y}{d t}=v_{k} \\
& \frac{d z}{d t}=w_{k}
\end{aligned}
$$

where are:

$$
\begin{aligned}
& E=\frac{\rho V^{2}}{2} \frac{S}{m}=\rho \frac{\pi d^{2}}{8 m} V^{2}, \\
& V=\sqrt{\left(u_{k}-u_{w}\right)^{2}+v_{k}^{2}+\left(w_{k}-w_{w}\right)^{2}}, \\
& \tilde{\beta}_{p}=-\frac{2 I_{x}}{\rho S d} \frac{g \cos \gamma}{V^{3}} \frac{p}{C_{\bar{m} \sigma}} .
\end{aligned}
$$

- aerodynamic functions $C_{D}(M a), C_{L \sigma}(M a)$, $C_{\bar{m} \sigma}(M a)$ and $C_{\ell p}(M a)$.

- constants $d, m$ and $I_{X}$,

- data on the state of the atmosphere $\rho(y), a(y)$ and data on wind $u_{w}(y), w_{w}(y)$,

- initial conditions $y_{0}$ (always take $x_{0}=z_{0}=0$ ), $V_{k 0}, \gamma_{k 0}, \chi_{k 0}$, and $p_{0}$.

- angular speed of rotation of the Earth

$\left[\begin{array}{l}\Omega_{E X} \\ \Omega_{E Y} \\ \Omega_{E Z}\end{array}\right]=\left[\begin{array}{c}\Omega_{E} \cos A_{0} \cos \varphi_{0} \\ \Omega_{E} \sin \varphi_{0} \\ -\Omega_{E} \sin A_{0} \cos \varphi_{0}\end{array}\right]$.

\section{FILE CALCULATION RESULTS „OJLMMT.IZL”}

TESMMT software solution is designed for testing and testing OJLMMT subroutine to calculate the path of classical stabilized projectile by the method of modified material point. Software solution is made in the programming language FORTRAN on a personal computer. It consists of three parts $[9,10]$ :
1. Program TESMMT.FOR - the main program,

2. File OJLMMT.DAT - input data,

3. File OJLMMT.IZL - calculation results.

Baseline data files,, OJLMMT.DAT”

$$
\begin{aligned}
& .153,43.56, .0584, .169, .1558,1 ., 1,2 \\
& 1000 ., 288.9, .0, .0 \\
& .0, .0, .0, .0, .0 \\
& .826,810, ., 01, .0, .0
\end{aligned}
$$

Figure 4 shows changes in the $\mathrm{Y}$-axis vertices trajectories of projectiles at different distances $\mathrm{X}$.

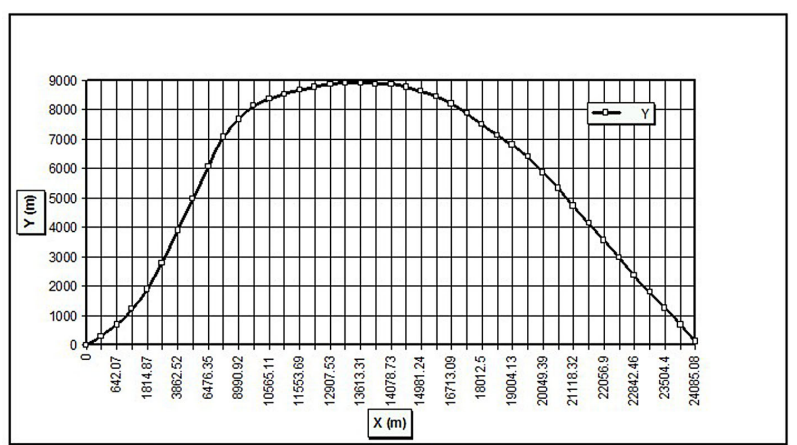

Figure 4. Change ordinate the vertices of $Y$ for different distance $\mathrm{X}$.

Figure 5 shows the change in the trajectories $\mathrm{Z}$ direction $(\mathrm{X})$.

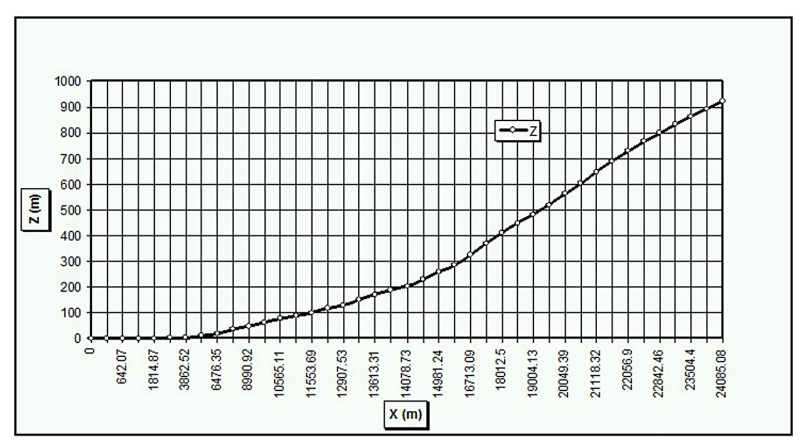

Figure 5. Change the direction $\mathrm{Z}(\mathrm{X})$ in the path of the projectile.

Figure 6 shows the change of the angular velocity for different distance shooting missiles $\mathrm{X}$. 


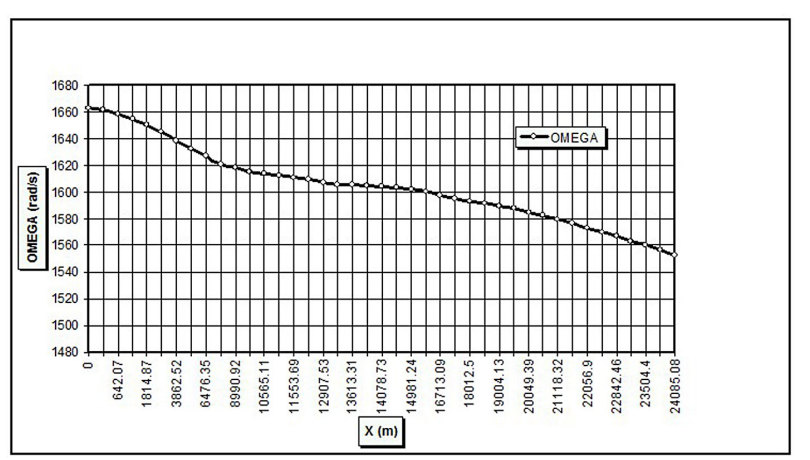

Figure 6. Change the angular velocity at different ranges of missiles.

Figure 7 shows the change in speed $\mathrm{V}$ of the trajectories of projectiles $\mathrm{X}$.

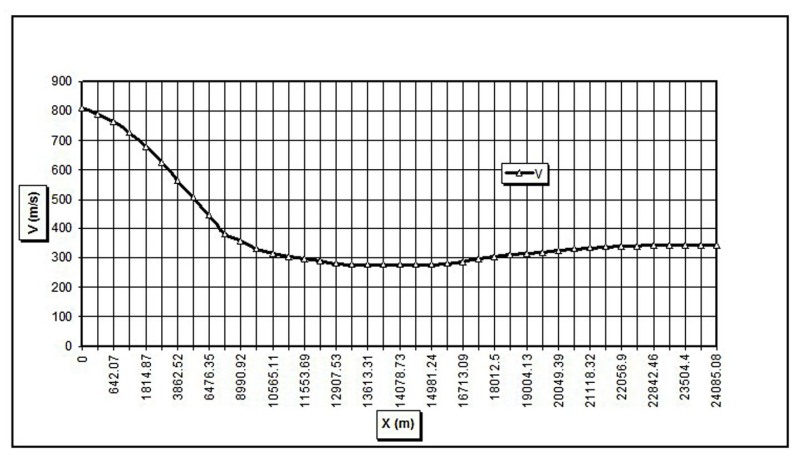

Figure 7. Change the speed V (X) at different distances

Figure 8 shows the change of angles $\alpha(\mathrm{X})$ and $\beta(\mathrm{X})$ at various distance learning shooting.

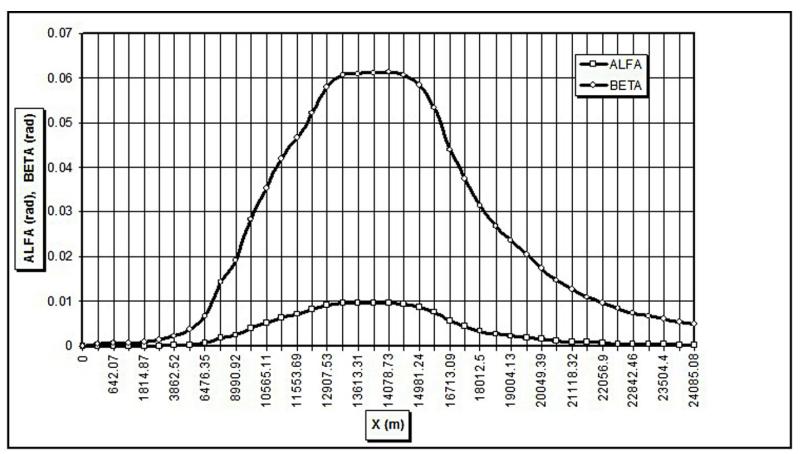

Figure 8. Changing the angle $\alpha(\mathrm{X})$ and $\beta(\mathrm{X})$ at different shooting distances.

Figure 9 shows the change of angles $\alpha(\beta)$ in the path of the projectile.

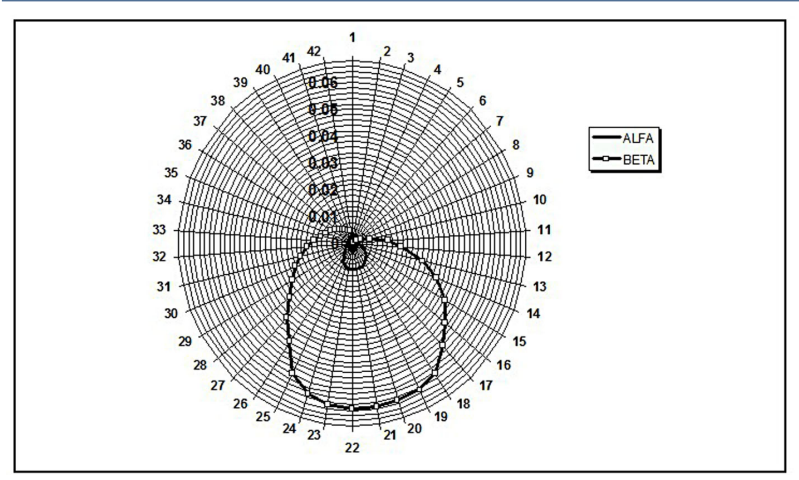

Figure 9. Changing the angle $\alpha(\beta)$ in the path of the projectile

\section{CONCLUSION}

When defining a physical model, it is necessary to first set the mathematical model and determine the missile. The six-degrees of freedom equations are the starting point for the modified point mass equations of motion. During the definition of a physical model neglected the effects of certain aerodynamic forces and moments to simplify calculations. Calculation of path elements is done by integrating the differential equations of motion modified material point method of Runge-Kutta fourth order. Such a composed software solution is universal and can be used for all tools that use defined projectile. For external ballistics calculation of the new, desired projectile is necessary to modify the input file or create a new defined-aerodynamic coefficients in subroutine AERO and baseline data of the selected missile. The output file is created during program executionand and the information obtained via WRITE command execution program is stored in it. These are external ballistics calculations. The program is organized so that the calculation stops at a given range of $\mathrm{x}$ and $\mathrm{y}$ axis. For standard atmosphere is carried out calculations of airspeed and Mach number of missiles in the current weather conditions at different altitudes projectile flight. Subroutine AERO define the aerodynamic coefficients depending on the current airspeed and Mach number. Special subroutine to solve systems of linear equations of the type $A x=b$. The calculation results show that the largest angle at the vertex orbit is around 0.062 radians. The fact that it satisfies the condition of stability for the most unfavorable situation means that the projectile is stable throughout the trajectory.

The proposed solution providing accurate calculations of the characteristics of the missile, the elements of stability and the conditions under which it is exercised. 
Applying software solutions proposed by the authors to save resources and reduce time numerical calculations as designing modern technical systems requires the use of simple physical and mathematical models and software that provide the required accuracy. The use of this software solution is in line with trends in the development of modern solutions in the external ballistics. This model allows the numerical integration of the equations for a shorter time than the model with six degrees of freedom.

\section{REFERENCES}

[1] Regodić D. (2003). Collection of solved problems from the external ballistics. Military Academy, Belgrade: MoD, Military Academy.

[2] Regodić D. (2006). External Ballistics. Belgrade: MoD, Military Academy.

[3] Krasnov N.F. (1985). Aerodinamics 1, 2. Moscow: Mir Publishers.

[4] McCormick, B. (1995), Aerodynamics, Aeronautics and Flight Mechanics. New York: John Wiley \& Sons, Inc.

[5] Carbonaro M. (1994). VKI Lecture Series Measurement Techniques inFluid Dynamics an Introduction. Rhode Saint Genèse -Belgium: Von Karman Institute for Fluid Dynamics Chaussée de Waterloo, 72 B - 1640 .

[6] Schmidt, V. Luis. (1998). Introduction to Aircraft Flight Dynamics. Washington: Education Series AIAA.

[7] Vinh, N. X. (1995). Flight Mechanics of High Performance Aircraft. Cambridge: University Press.

[8] Pamadi, B. N. (1998). Performance, Stability, Dynamics and Control of Airplanes. Washington: Education Series AIAA.
[9] McCoy R.L. (1999). Modern Exterior Ballistics. The Launch and Flight Dynamics of Symmetric Projectiles, Atglen, Pennsylvania, USA: Schiffer Publishing.

[10] Raymer, D. (1992). Aircraft Design: A Conceptual Approach, Washington: AIAA Education Series.

[11] Mair, W.A. and Birdsall, D. (1992), Aircraft Performance, Cambridge: University Press.

[12] Robert F. Lieske, Robert F. McCoy (1964), Equations of motion of ARIGID body, Maryland, USA: Ballistics research Laboratories.

[13] Jerkovic, D., Regodic D. (2011). The influence of aerodynamic coefficients on the elements of classic projectile paths. Military Technical Courier, LIX, 2/2011, pp. 5-28

[14] ISO Concepts. (1988). Quantities and Symbols for Flight Dynamics, Part 1: Aircraft motion relative to the air, ISO/DIS 1151/1, and Part 2: Motion of the aircraft and the atmosphere relative to the Earth, ISO/DIS 1151/2

[15] Murphy, C.H. (1963). Free Flight Motion of Symmetric Missiles, Ballistic Resarch Laboratories Report No.1216.

[16] McCoy, R. L. (1985). Aerodynamic and Flight Dynamic Characteristics of the New Family of 5.56 mm NATO Ammunition, Ballistic Research Laboratory Memorandum Report No. BRL-MR-3476.

[17] McCoy, R. L. (1990). The Aerodynamic Characteristics of .50 Ball, M33, API, M8, and APIT, M20Ammunition. Ballistic Research Laboratory Memorandum Report No. BRL-MR-3810.

[18] McCoy, R.L. (1992).The Effect of Yaw Cards on the Pitching and Yawing Motion of Symmetric Projectiles. Ballistic Research Laboratory Technical Report BRL-TR-3338.

[19] Piddington,M.J. (1967). Aerodynamic Characteristics of the $7.62 \mathrm{~mm}$ NATO Ammunition M-59, M-80, M-61, M-62, Ballistic Research Laboratory memorandum Report No. 1833. 